\title{
Considering prevision driving behavior in car-following model
}

\author{
HU Junjun ${ }^{1, a}$,ZHANG Yu ${ }^{2, b}$ and ZHAO Ruiyu ${ }^{1, c}$ \\ ${ }^{1}$ College of Mobile Telecommunications, Chongqing University of Posts and Telecom, Chongqing \\ 401520, China \\ ${ }^{2}$ Chongqing ChuanYi Automation Co. Ltd., Chongqing 401121, China \\ aesp_hi@163.com, ${ }^{b}$ kaixinyuzhangyu@163.com, ${ }^{c}$ 273650674@qq.com
}

Keywords: traffic flow; car-following model; prevision driving behavior; traffic jam

Abstract. In the paper, we develop a new car-following model considering the prevision driving behavior on a single-lane road. The model's linear stability condition is obtained by applying the linear stability theory. And through nonlinear analysis, a modified Korteweg-de Vries (mKdV) equation is derived to describe the propagating behavior of traffic density wave near the critical point. Numerical simulation shows that the new model can improve the stability of traffic flow by adjusting the driver's prevision intensity parameter, which is consistent with the theoretical analysis.

\section{Introduction}

Traffic jam has been a serious problem in modern city traffic and many traffic models[1-4], such as car-following models, cellular automaton models, gas kinetic models, and hydrodynamic models, have been developed to investigate the properties of traffic jams. The optimal velocity $(\mathrm{OV})$ model proposed by Bando et al. [5], one of the favorable car-following traffic models on studying traffic flow, has successfully described the formation of traffic jams and revealed the transition mechanism in a simple way. Subsequently, many works have been done based on the OV model [6,7].

These car-following models mentioned above can reproduce many complex actual traffic phenomena, but they can not be employed to study the influence of the prevision driving behavior since they did not consider the factor. In fact, a leading car's driver can send the future speed information of the leading car to the follower before the leading car's the current speed changes by using the intelligent transportation system (ITS). In this way, the following car's driver can control his current speed in advance to achieve the optimal state. However, until now the car-following model with considering the prevision driving behavior has not been explored so far.

In this paper, a new car following model is proposed by taking the prevision driving behavior on a single-lane road into account. In the following section, the new car following model is introduced. In Section 3, linear stability analysis is conducted. In Section 4, nonlinear analysis is done. In Section 5, numerical simulation is carried out to validate the analytic results. Conclusions are given in Section 6.

\section{Models}

In 1995, Bando et al. [5] proposed the OV model to describe the car-following behavior on a single lane highway. The motion equation is given as follows:

$$
d v_{j}(t) / d t=a\left[V\left(\Delta x_{j}(t)\right)-v_{j}(t)\right]
$$

where $x_{j}(t)$ is the position of car at $j$ time $t, \Delta x_{j}(t)=x_{j+1}-x_{j}$ represents the headway of two successive vehicles, $a$ is the sensitivity of a driver, and $V\left(\Delta x_{j}(t)\right)$ is the optimal velocity function. The comparison with empirical data shows that the OV model appears too high acceleration and unrealistic deceleration.

In order to overcome the deficiency, Helbing and Tilch [8] proposed a generalized force (GF) model, i.e.

$$
d v_{j}(t) / d t=a\left[V\left(\Delta x_{j}(t)\right)-v_{j}(t)\right]+\lambda H\left(-\Delta v_{j}(t)\right) \Delta v_{j}(t)
$$


where $H$ is the Heaviside function, $\lambda$ is a sensitivity coefficient different from $a, \Delta v_{j}(t)=v_{j+1}-v_{j}$ is the velocity difference between the leading car $j+1$ and the following car $j$. The simulation results indicate that the GF model is poor in anticipating the kinematics wave speed and the delay time of car motion.

In 2001, by introducing the positive relative velocity into the GF model, Jiang et al.[9] developed full velocity difference (FVD) model as follows:

$$
d v_{j}(t) / d t=a\left[V\left(\Delta x_{j}(t)\right)-v_{j}(t)\right]+\lambda \Delta v_{j}(t)
$$

The results illustrate that FVD model has better agreement with the field data than the OV and GF model.

The aforementioned models can describe some complex traffic phenomena (e.g, congestion, instability and stop-and-go waves in traffic flow). However, these models are unsuited to study the prevision driving behavior since they doesn't consider it at all. In fact, by applying ITS, a driver adjusts his current velocity in advance based on the dynamic prevision velocity information of the leading one, i.e., whether the car will decelerate or accelerate in advance, or just motion at the current velocity. In view of the above reason, we develop a new prevision driving car-following (for short, PD-CF) model with considering the prevision driving behavior, whose dynamics equation is

$$
d v_{j}(t) / d t=a\left\{V\left(\Delta x_{j}(t)\right)-v_{j}(t)\right\}+\lambda \Delta v_{j}(t)+k\left(v_{j+1}(t+1)-v_{j+1}(t)\right)
$$

where $\Delta F_{j+1}=v_{j+1}(t+1)-v_{j+1}(t)$ represents the velocity difference between the future speed and the current speed of the leading car $j+1$. FVD model did not take the velocity difference term into consideration, however, we consider that term impact the traffic stability, and thus it is introduced in our model by response factor $k$. When $k=0$, Eqs.(4) of the extended model reduce into those of FVD model. For later convenience of simulation and nonlinear analysis, Eq. (4) can be rewritten in terms of the headway:

$$
\begin{aligned}
& \Delta x_{j}(t+2 \tau)=\Delta x_{j}(t+\tau)+\tau\left[V\left(\Delta x_{j+1}(t)\right)-V\left(\Delta x_{j}(t)\right)-\Delta v_{j}(t)\right] \\
& +\tau \lambda\left[\Delta v_{j+1}(t)-\Delta v_{j}(t)\right]+\tau k\left[v_{j+2}(t+1)-2 v_{j+1}(t+1)+v_{j+1}(t)\right]
\end{aligned}
$$

\section{Linear stability analysis}

In order to investigate the impact of the prevision driving behavior on the traffic flow, the linear stability analysis can be conducted for PD-CF model. The vehicles move with the uniform headway $b$ and the optimal velocity $V(b)$. Therefore, the steady-state solution is given as

$$
x_{j}^{0}(t)=b j+V(b) t, b=L / N
$$

where $L$ is the road length and $N$ is the car number. Suppose $y_{j}(t)$ is a small deviation from the steady state:

$$
x_{j}(t)=x_{j}^{0}(t)+y_{j}(t)
$$

Substituting Eq.(7) into Eq.(5) and linearizing the resulting equation, we can obtain

$$
y_{j}^{\prime \prime}(t)=a\left[V^{\prime}(b) \Delta y_{j}(t)-y_{j}^{\prime}(t)\right]+\lambda \Delta y_{j}^{\prime}(t)+\frac{k}{2} y_{j+1}^{\prime \prime}(t)
$$

where $V^{\prime}=d V\left(\Delta x_{j}\right) /\left.d \Delta x_{j}\right|_{\Delta x_{j}=b}$. By expanding $y_{j}(t)=e^{i k j+z t}$, we obtain the following equation for $z$

$$
z^{2}+\left(a-\lambda e^{k i}+\lambda\right) z-a V^{\prime}(b)\left(e^{k i}-1\right)-\frac{k}{2} e^{k i}=0
$$

Inserting $z=z_{1} i k+z_{2}(i k)^{2}+\mathrm{L}$ into Eq. (9) and neglecting the higher order terms, then the first order and second order terms of $i k$ are obtained as follows: $z_{1}=V^{\prime} \quad$ and $\quad z_{2}=\left(V^{\prime}+\lambda+\frac{k}{2}\right) / a-1 / 2$. Thus the neutral stable criterion is: 


$$
V^{\prime}=\frac{1}{2} a+\lambda+\frac{k}{2}
$$

For small disturbance with long wavelengths, the uniform traffic flow is stable if

$$
V^{\prime}<\frac{1}{2} a+\lambda+\frac{k}{2}
$$

Fig. 1 shows the neutral stable curves in the headway-sensitivity space ( $\Delta x, a)$ for PD-CF model under different values of $k$. The apex of each curve indicates the critical point $\left(h_{c}, a_{c}\right)$. Above the neutral stability line, the traffic flow is stable and traffic jams will not appear. While below the line, traffic flow is unstable and the density waves emerge. From Fig. 1 one can find that with the increase of $k$ the stability region is gradually enlarged, which indicates that the prevision driving effect plays an important role in enhancing the stability of traffic flow. As $k=0$, the neutral stability line is the same as that of FVD model.

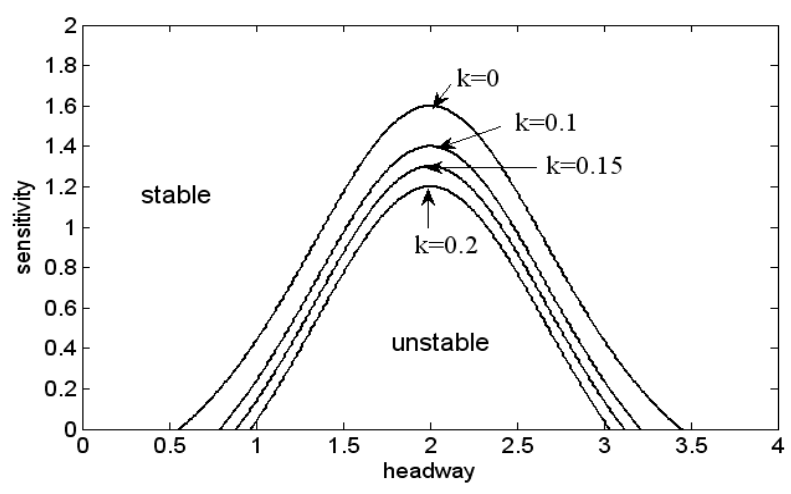

Fig.1 Phase diagram in headway-sensitivity space $(\Delta x, a)$.

\section{Numerical simulation}

Computer simulation is carried out to check the validity of our theoretical results above. Under the periodic boundary condition, the following initial conditions are chosen as follows: $\Delta x_{j}(0)=\Delta x_{j}(1)=4.0$, for $j \neq 50,51, \Delta x_{j}(1)=4.0+0.1$, for $j=50, \Delta x_{j}(1)=4.0-0.1$, for $j=51$.

The total number of cars is $\mathrm{N}=100$ and the sensitivity $a=1.4$.

Fig. 2 shows the space-time evolution of the headway after $t=10^{4}$ time steps under the different parameter $k$. The patterns (a)-(d) in Fig. 2 exhibit the time evolution of the headway for $k=0,0.1,0.15$ and 0.2 respectively. Pattern (a) with $k=0$ corresponds to that of the FVD model. In patterns (a) and (b), it can be seen clearly the traffic flow are unstable and the kink-antikink soliton appears because the linear instability condition (11) is unsatisfied at $a=1.4$. When a small disturbance is added into the uniform traffic flow, the propagating backward stop-and-go traffic jam appears which is very similar to the mKdV solution. However, comparing patterns (a) with (b) with the same sensitivity, one can find that the traffic congestion is much less serious in pattern (b), which means that the new consideration plays the positive function on the stabilization of traffic flow. As increasing the parameter $k$, from pattern (b)-(d), the amplitude of the kink-antikink soliton weakens gradually. If we set $k=0.2$, due to the stability condition is satisfied, the stop-and-go phenomenon disappears and traffic flow turns uniform over the whole space in pattern (d) again, which further demonstrates that the prevision driving behavior can enhance the stability of traffic flow.

\section{Conclusions}

A new car following model considering the prevision driving behavior is developed and it has been analyzed by using the linear stability theory and the nonlinear analysis. The stability condition of 


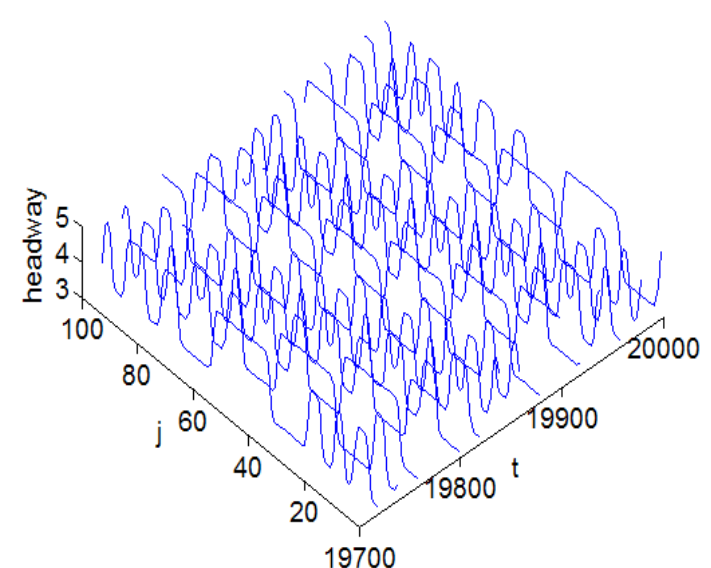

(a)

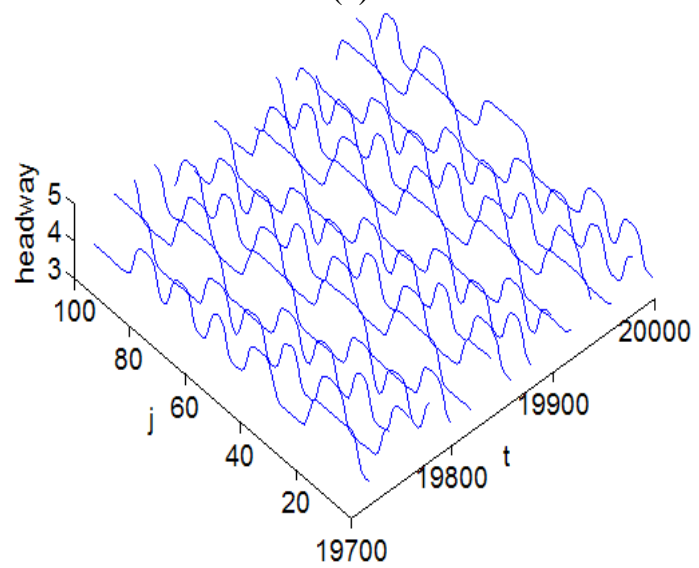

(c)

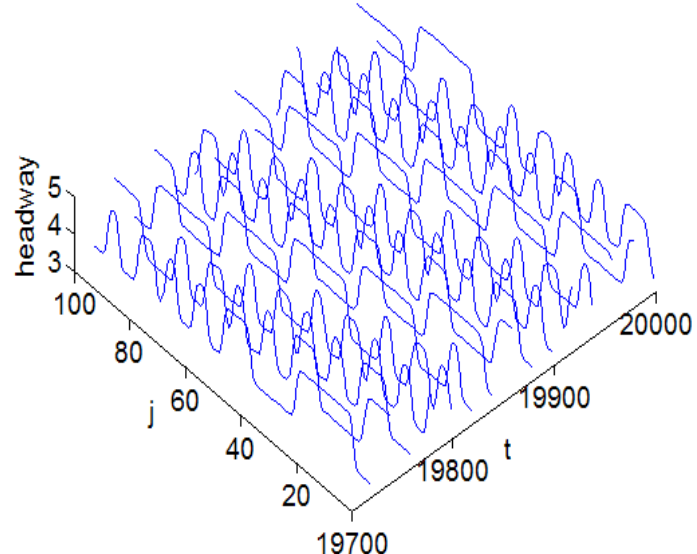

(b)

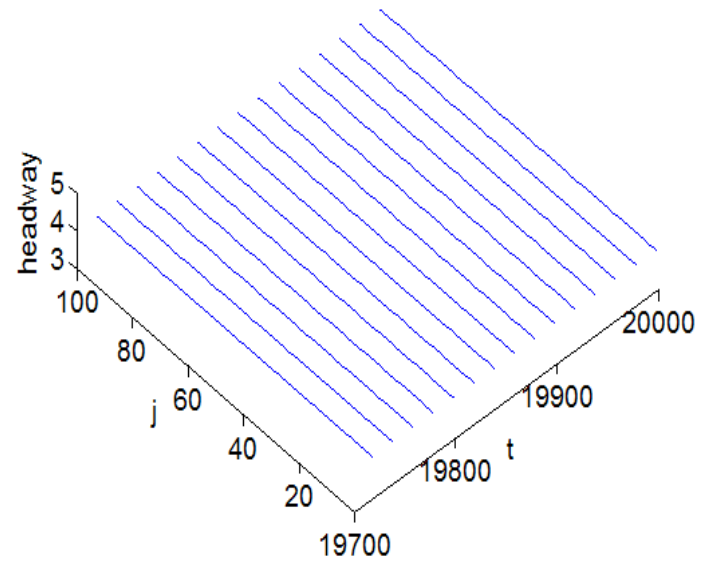

(d)

Fig.2 Space-time evolution of the headways after $t=10000$ under the different value of $k$ the extended model is obtained and the results show that the stability of traffic flow is improved by taking driver's prevision effect into account. Moreover, The kink-antikink soliton solution of mKdV equation near the critical point is derived to describe the traffic jams. Numerical simulation shows that the traffic jams are suppressed efficiently by considering prevision driving behavior. The analytical results are in good agreement with the simulation results.

\section{References}

[1] Y. F. Jin, H. Y. Hu. Stabilization of traff flow in optimal velocity model via delayed-feedback control: Commun Nonlinear Sci Numer Simul Vol.18 (2013), p.1027-34.

[2] S. Jin, D. H. Wang, P. F. Tao, P. F. Li. Non-lane-based full velocity difference car following model:Physica A Vol. 389(2010), p.4654-4662 .

[3] C. Tian, D. H. Sun, M. Zhang. Nonlinear analysis of lattice model with consideration of optimal current difference: Commun Nonlinear Sci Numer Simul 16 Vol. (2011), p.4524-29.

[4] T. Q. Tang, H. J. Huang, H. Y. Shang. A new macro model for traffic flow with the consideration of the driver's forecast effect: Phys Lett A Vol. 374 (2010) , p.1668-1672.

[5] M. Bando, K. Hasebe, A. Nakayama, A. Shibata, Y. Sugiyama. Dynamical model of traffic congestion and numerical simulation: Phys Rev E Vol.51(1995), p. 1035-42.

[6] G. Guo, W. Yue. Hierarchical platoon control with heterogeneous information feedback: IET Control Theory \& Applications Vol. 5(2011), p.1766-1781.

[7] C. Y. Li, T. Q. Tang, H. J. Huang, H. Y. Shang. A New Car-Following Model with Consideration of Driving Resistance: Chin. Phys.Lett Vol. 28(2011), p. 038902.

[8] D. Helbing, B. Tilch. Generalized force model of traffic dynamics: Phys Rev E, Vol. 58(1998), p.133-138.

[9] R. Jiang, Q. S. Wu, Z. J. Zhu. Full velocity difference model for a car-following theory:Phys Rev E Vol. 64(2001), p.017101-017104. 